\title{
Redox induces diverse effects on recombinant human wild-type PrP and mutated PrP with inserted or deleted octarepeats
}

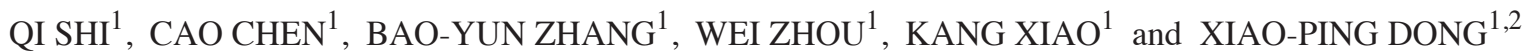 \\ ${ }^{1}$ State Key Laboratory for Infectious Disease Prevention and Control, Collaborative Innovation Center for Diagnosis and \\ Treatment of Infectious Diseases (Zhejiang University, Hangzhou), National Institute for Viral Disease Control and Prevention, \\ Chinese Center for Disease Control and Prevention, Beijing 102206; ${ }^{2}$ Chinese Academy of Sciences Key Laboratory of \\ Pathogenic Microbiology and Immunology, Institute of Microbiology, \\ Chinese Academy of Sciences, Beijing 100101, P.R. China
}

Received February 29, 2016; Accepted November 30, 2017

DOI: $10.3892 /$ ijmm.2018.3441

\begin{abstract}
Normal prion protein (PrP) contains two cysteines at amino acids 179 and 214, which may form intra- and interpeptide disulfide bonds. To determine the possible effects of this disulfide bridge on the biochemical features of PrP, prokaryotic recombinant human wild-type PrP (PG5), and mutated PrPs with seven extra octarepeats (PG12) or with all five octarepeats removed (PG0), were subjected to redox in vitro. Sedimentation assays revealed a large portion of aggregation in redox-treated PG5, but not in PG0 and PG12. Circular dichroism analysis detected increased $\beta$-sheet and decreased $\alpha$-helix in PG5 subjected to redox, increased random-coil and decreased $\beta$-sheet in PG0, and increased random-coil, but limited changes to $\beta$-sheet content, in PG12. Thioflavin T fluorescence tests indicated that fluorescent value was increased in PG5 subjected to redox. In addition, proteinase K (PK) digestions indicated that PK resistance was stronger in PG12 and PG0 compared with in PG5; redox enhanced the PK resistance of all three PrP constructs, particularly PG0 and PG12. These data indicated that formation of a disulfide bond induces marked alterations in the secondary structure and biochemical characteristics of PrP. In addition, the octarepeat region within the PrP peptide markedly influences the effects of redox on the biochemical phenotypes of PrP, thus highlighting the importance of the number of octarepeats in the biological functions of PrP.
\end{abstract}

Correspondence to: Professor Xiao-Ping Dong, State Key Laboratory for Infectious Disease Prevention and Control, Collaborative Innovation Center for Diagnosis and Treatment of Infectious Diseases (Zhejiang University, Hangzhou), National Institute for Viral Disease Control and Prevention, Chinese Center for Disease Control and Prevention, 155 Chang-Bai Road, Beijing 102206, P.R. China

E-mail: dongxp238@sina.com

Key words: prion protein, redox, disulfide bond, octarepeat, thioflavin $\mathrm{T}$

\section{Introduction}

Prions are infectious agents without detectable nucleic acids, which cause prion diseases or transmissible spongiform encephalopathies (TSEs) in humans and various animal species (1). The conversion from a normal, endogenous membrane glycoprotein [cellular prion protein $\left(\operatorname{PrP}^{\mathrm{C}}\right)$ ] to a pathological, conformationally altered isoform [scrapie prion protein $\left(\mathrm{PrP}^{\mathrm{Sc}}\right)$ ] is the crux of prion biology. The most notable alteration in the secondary structure during the conversion from $\operatorname{PrP}^{\mathrm{C}}$ to $\operatorname{PrP}^{\mathrm{Sc}}$ is a decrease in its $\alpha$-helix content and a marked increase in $\beta$-sheet content. Besides its infectivity in homologous, and some heterologous species, $\mathrm{PrP}^{\mathrm{Sc}}$ displays distinct biochemical characteristics, including insoluble, easily formed aggregates or fibrils, and partial resistance to proteolytic digestion (2).

$\mathrm{PrP}$ is a conserved protein among mammals. Full-length $\mathrm{PrP}$ is 253-254 amino acids (aa) long, with a signal peptide (1-22 aa) in the N-terminus and a glycosylphosphatidylinositol anchor (232-254 aa) in the C-terminus. In the N-terminus, there is an octarepeat region that consists of five octapeptides, which exert various biological activities, including binding with copper, manganese and zinc metal ions, promoting PrP internalization and interacting with other proteins, such as glial fibrillary acidic protein and tubulin (3). Naturally occurring insertions or deletions of octapeptide repeats in this region are associated with the human prion disease, genetic or familial Creutzfeldt-Jacob disease. In addition, insertions or deletions of octapeptide repeats have been confirmed to induce similar pathogenicity in rodent animals $(4,5)$. PrP contains two N-linked glycosylation sites at asparagine (Asn)181 and Asn197, which contribute to the formation of three PrP isoforms: Di- mono- and unglycosylated PrP, in host cells (6). N-linked glycan helps the folding process of newly synthesized protein, whereas removal of PrP glycosylation provokes cell apoptosis (7). Transgenic mice bearing a glycosylation deficiency at either of the two glycan attachment sites of $\mathrm{PrP}^{\mathrm{C}}$ exhibit increased sensitivity to bovine spongiform encephalopathy and scrapie. In addition, a higher ratio of unglycosylated PrP has been repeatedly detected in the brain tissues of humans and animals with TSEs (8). These findings indicate the importance of the maintenance of aa sequences and post-translational modification of PrP protein. 
In the C-terminal fragment of PrP, there are two cysteines (Cys) at aa 179 and 214, which are critical for forming intra- and/or intermolecular disulfide bridges (9). Formation of a disulfide bond serves an important role in protein folding, stabilizing protein conformational structure and biological function $(10,11)$. In the present study, three prokaryotic human PrP constructs: Wild-type PrP (PG5), mutant PrP with insertion of seven extra octarepeats (PG12) and mutant PrP with deletion of all five octarepeats (PG0), underwent redox in vitro, in order to form a disulfide bond. Subsequently, the biochemical features of these PrP proteins were evaluated.

\section{Materials and methods}

Plasmids. The generation of the following recombinant prokaryotic protein-expressing plasmids: pQE30-huPrP23-231 containing five octapeptide repeats (wild-type PrP, PG5), pQE30-huPG12 containing 12 octapeptide repeats (PG12) and pQE30-huPG0 with deletion of all five octapeptide repeats (PG0) was described previously (12).

Protein purification. The bacterially expressed recombinant polyhistidine (His)-tagged PrPs were expressed and purified according to previously described protocols (13). Briefly, the expressed PrPs were recovered from urea-solubilized bacterial lysate following sonication by immobilized nickel-based affinity chromatography. The final protein product was $>95 \%$ pure and was concentrated to $0.6 \mathrm{mg} / \mathrm{ml}$ with $10 \mathrm{mM} \mathrm{NaAC}$. Proteins were aliquoted and stored at $-80^{\circ} \mathrm{C}$ for further analyses. Protein concentration of the dissolved product was determined in triplicate using the bicinchoninic acid (BCA) protein assay kit (Thermo Fisher Scientific, Inc., Waltham, MA, USA) according to the manufacturer's protocol.

Redox of recombinant PrPs. Reduction of the purified PrPs was conducted based on a previously described protocol, with modifications (14). The various PrPs (PG0, PG5 and PG12) were initially incubated in redox buffer A $(50 \mathrm{mM}$ Tris- $\mathrm{HCl}, 100 \mathrm{mM}$ DTT, 2.5 M GdnHCl, $3 \mathrm{M} \mathrm{NaCl}$, pH 8.0) at $37^{\circ} \mathrm{C}$ for $16 \mathrm{~h}$, in order to produce thiol groups and free radicals. Subsequently, the proteins were incubated with redox buffer B $(150 \mathrm{mM}$ $\mathrm{ICH}_{2} \mathrm{CONH}_{2}, 50 \mathrm{mM}$ DTT, $500 \mathrm{mM}$ Tris- $\left.\mathrm{HCl}, \mathrm{pH} 8.5\right)$ at $37^{\circ} \mathrm{C}$ for $6 \mathrm{~h}$, in order to produce alkylation of thiol groups to protect the free thiol groups. Proteins were then carefully dialyzed in dialysis buffer A (10 mM NaAc, $50 \mathrm{mM} \mathrm{NaCl}, \mathrm{pH} 4.0)$ and buffer B (10 mM NaAc, pH 5.0), respectively, to form disulfide bonds via oxidation of thiol groups.

Sedimentation experiments. Samples were centrifuged for $30 \mathrm{~min}$ at 20,000 $\mathrm{xg}$ at $4^{\circ} \mathrm{C}$. The supernatants and pellets were separately collected and the protein pellets were resuspended in a volume equal to the volume of the supernatants. The fractions were separated by $12 \%$ SDS-PAGE and were then analyzed by Commassie blue staining.

Circular dichroism (CD) analysis. Various samples of redox recombinant PrPs were dissolved in $1 \mathrm{mM}$ MES solution ( $\mathrm{pH}$ 5.5) at $10 \mu \mathrm{M}$ final concentration. Three samples from each recombinant $\operatorname{PrP}$ solution were independently evaluated by $\mathrm{CD}$ analysis. CD spectra were recorded in a $0.1 \mathrm{~cm}$ path length quartz cell at room temperature under constant nitrogen flush using a Jasco J720 spectropolarimeter (JASCO, Easton, MD, USA). Subsequently, three spectra were accumulated and the appropriate blanks were subtracted. The values were expressed as molar ellipticity $(\theta)$. Estimates of percentage secondary structure were obtained using $\mathrm{K} 2 \mathrm{D}$ analysis programs.

Thioflavin $T$ (ThT) fluorescence. PrPs that did or did not undergo redox were incubated in assembly buffer containing $50 \mathrm{mM}$ Tris- $\mathrm{Cl}$ (pH 7.4), $150 \mathrm{mM} \mathrm{KCl}$ and $10 \mathrm{mM}$ ATP at $37^{\circ} \mathrm{C}$ for various durations. For ThT assays, $10 \mu \mathrm{l}(0.6 \mu \mathrm{g})$ of each preparation was mixed with ThT solution $(180 \mu \mathrm{l})$ containing $50 \mathrm{mM}$ glycine- $\mathrm{OH}(\mathrm{pH} 8.5)$ and $5 \mu \mathrm{M}$ ThT at room temperature for $1 \mathrm{~min}$ ( $1 \mathrm{mM}$ stock solution in water; Sigma T3516; Sigma-Aldrich; Merck KGaA, Darmstadt, Germany). The fluorescence of each sample was measured using a spectropolarimeter (F-4500; Hitachi, Ltd., Tokyo, Japan) at $485 \mathrm{~nm}$ using an excitation wavelength of $440 \mathrm{~nm}$.

Proteinase $K(P K)$ resistance assay. Recombinant PrPs that did or did not undergo redox were assessed for PK sensitivity following treatment with 6.25, 12.5 and $25 \mu \mathrm{g} / \mathrm{ml}$ PK (Roche Diagnostics $\mathrm{GmbH}$, Mannheim, Germany) at $37^{\circ} \mathrm{C}$ for $20 \mathrm{~min}$ or $1 \mathrm{~h}$. The reaction was terminated by boiling for $5 \mathrm{~min}$ with SDS-PAGE sample loading buffer.

Western blot analysis. Protein samples were separated by $12 \%$ SDS-PAGE and were electrotransferred onto nitrocellulose (NC) membranes. All protein extracts were quantified using BCA reagent (Merck ,Kenilworth, NJ, USA) prior to resolution with SDS-PAGE and electrotransfer to NC membranes (Whatman; GE Healthcare Bio-Sciences, Pittsburgh, PA, USA). Membranes were blocked with $5 \%$ (w/v) bovine serum albumin in $1 \mathrm{X}$ Tris-buffered saline containing $0.1 \%$ Tween-20 (TBST) at room temperature for $2 \mathrm{~h}$ and were then probed with 1:5,000-diluted PrP specific monoclonal antibody 3F4 (MAB1562; EMD Millipore, Billerica, MA, USA) at $4^{\circ} \mathrm{C}$ overnight. After washing with TBST, membranes were subsequently incubated with 1:5,000-diluted goat anti-mouse secondary antibodies (cat. no. 32723; Thermo Fisher Scientific, Inc.) and reactive signals were visualized using an enhanced chemiluminescence kit (PE, Waltham,MA, USA). Images were captured using a ChemiDoc ${ }^{\mathrm{TM}}$ XRS+ Imager (Bio-Rad Laboratories, Inc., Hercules, CA, USA). Densitometric analysis of western blot analyses was conducted using ImageJ software version 1.44 (National Institutes of Health, Bethesda, MD, USA).

Statistical analysis. All of the experiments were performed at least three times, with consistent results. Statistical analysis was performed using GraphPad Prism6 Software (GraphPad Software, Inc., La Jolla, CA, USA). All data values are presented as the means \pm standard deviation . P-values for differences between two groups were determined by two-tailed t-test. $\mathrm{P}<0.05$ was considered to indicate a statistically significant difference.

\section{Results}

Effects of redox on the sedimentation features of three recombinant human PrPs. The expression of recombinant 

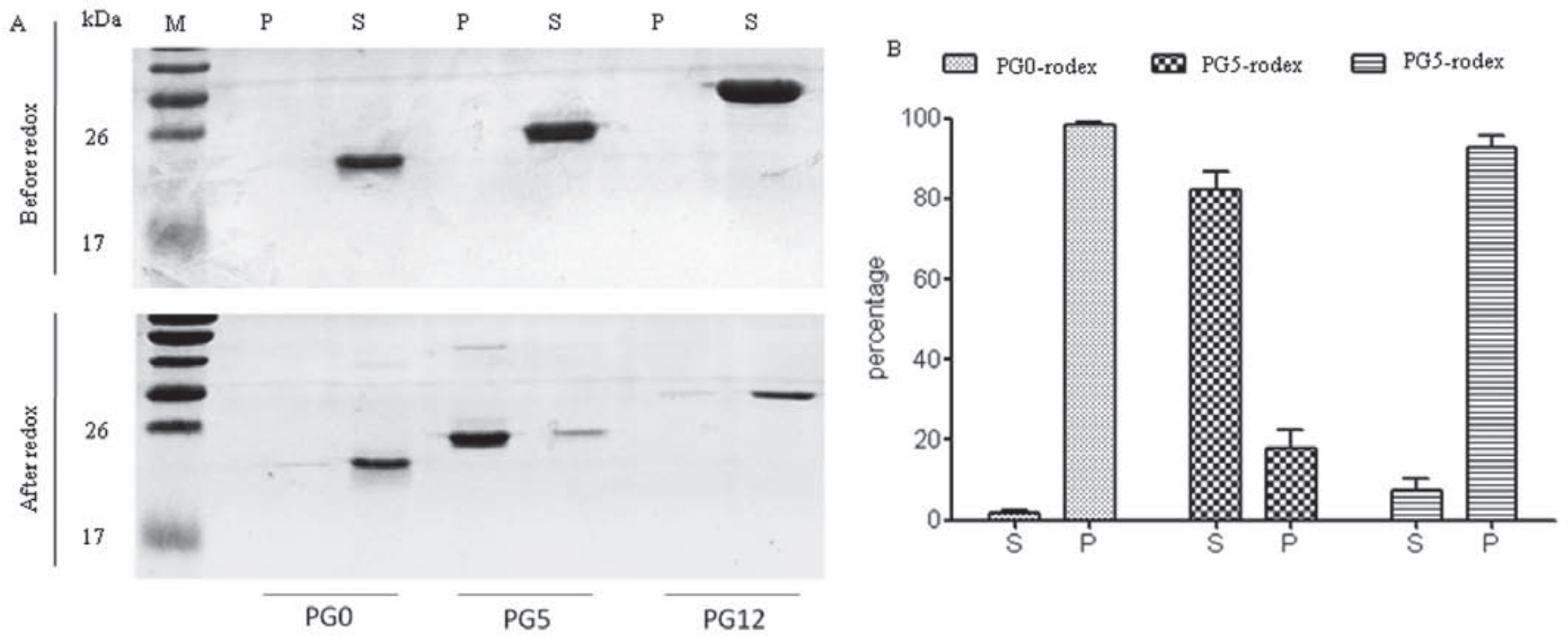

Figure 1. Effects of redox on the sedimentation features of PG0, PG5 and PG12. (A) Preparations were separated by $12 \%$ SDS-PAGE and were visualized by Commassie blue staining following centrifugation. Upper panel, prior to redox. Lower panel, after redox. (B) Semi-quantitative analyses of the average gray values of PrPs in the $\mathrm{P}$ and $\mathrm{S}$ fractions following redox. The average proportions of PrPs in the $\mathrm{P}$ and $\mathrm{S}$ fractions were calculated from three independent tests and are presented as the means \pm standard deviation. P, pellet; PrP, prion protein; S, supernatant.

human His-tagged PrPs (PG0, PG5 and PG12) was induced in Escherichia coli strain M15. SDS-PAGE of the purified products revealed that PG0 was 20-kDa, PG5 was 25-kDa and PG12 was 30-kDa; these proteins were specifically recognized by the PrP 3F4 monoclonal antibody by western blotting (data not shown). To determine the possible effects of redox on the sedimentation characteristics of these three PrP constructs, PG0, PG5 and PG12 preparations were centrifuged before and after redox. SDS-PAGE revealed that almost all PrPs were present in the supernatant fractions of all three PrP constructs prior to redox (Fig. 1A, upper panel). Notably, following redox, the majority of PG5 transferred to the pellet fraction, whereas PG0 and PG12 mutants remained in the supernatant fraction (Fig. 1A, lower panel). Densitometric analysis of the gray values of PrP protein bands demonstrated that $\sim 80 \%$ PG5 was present in the pellet following redox (Fig. 1B).

Effects of redox on the secondary structure of the three recombinant human PrPs. To determine the effects of redox on PrP structure, the secondary structures of PrP proteins were examined before and after redox by far-ultraviolet (UV) CD spectra. As shown in Fig. 2, redox-untreated PG5 and PG12 displayed two maximum UV absorbance peaks (at 210 and $222 \mathrm{~nm}$ ) representing $\alpha$-helix predominance, whereas redox-untreated PG0 exhibited a UV absorbance peak at $216 \mathrm{~nm}$, indicating $\beta$-sheet enrichment. Following redox, the two UV absorbance peaks in PG5 and PG12 disappeared. The contents of the main structures of the three PrP constructs prior to and following redox were evaluated and summarized in Table I. As expected, the $\alpha$-helix content of the three PrP constructs was markedly reduced by redox, particularly in PG5 and PG0. In addition, the $\beta$-sheet content was markedly increased in redox-treated PG5, whereas random-coil content was predominant in redox-treated PG0. In redox-treated PG12, there was a marked

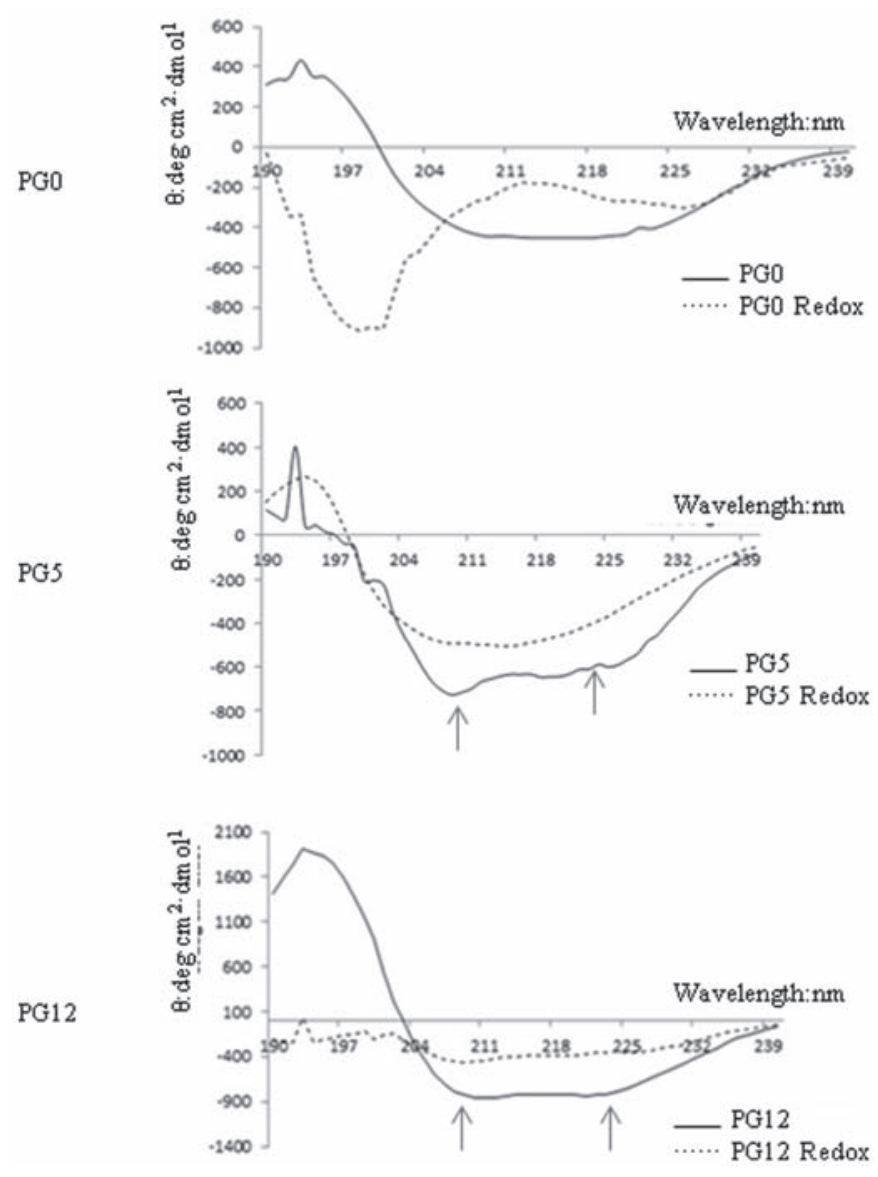

Figure 2. Far-UV CD spectral analysis of the secondary structures of PG0, PG5 and PG12 before and after redox. The thin dotted line represents PrPs following redox and the thick line represents PrPs prior to redox. Three independently performed spectra were accumulated and the values are expressed as molar ellipticity $(\theta)$ on the $y$-axis. Wavelength in $\mathrm{nm}$ is presented on the $\mathrm{x}$-axis. $\mathrm{CD}$, circular dichroism; PrP, prion protein; $\mathrm{UV}$, ultraviolet. 
Table I. $\alpha$-helix and $\beta$-sheet contents of three human prion protein constructs prior to and after redox.

\begin{tabular}{lcccccc}
\hline Structure & PG0 & PG0 redox & PG5 & PG5 redox & PG12 & PG12 redox \\
\hline$\alpha$-helix & 17.1 & 3.7 & 24.9 & 1.9 & 55.4 & 22.0 \\
$\beta$-sheet & 53.9 & 22.7 & 36.2 & 52.6 & 33.8 & 38.2 \\
Random-coil & 29.0 & 73.6 & 38.9 & 45.5 & 10.9 & 39.9 \\
\hline
\end{tabular}

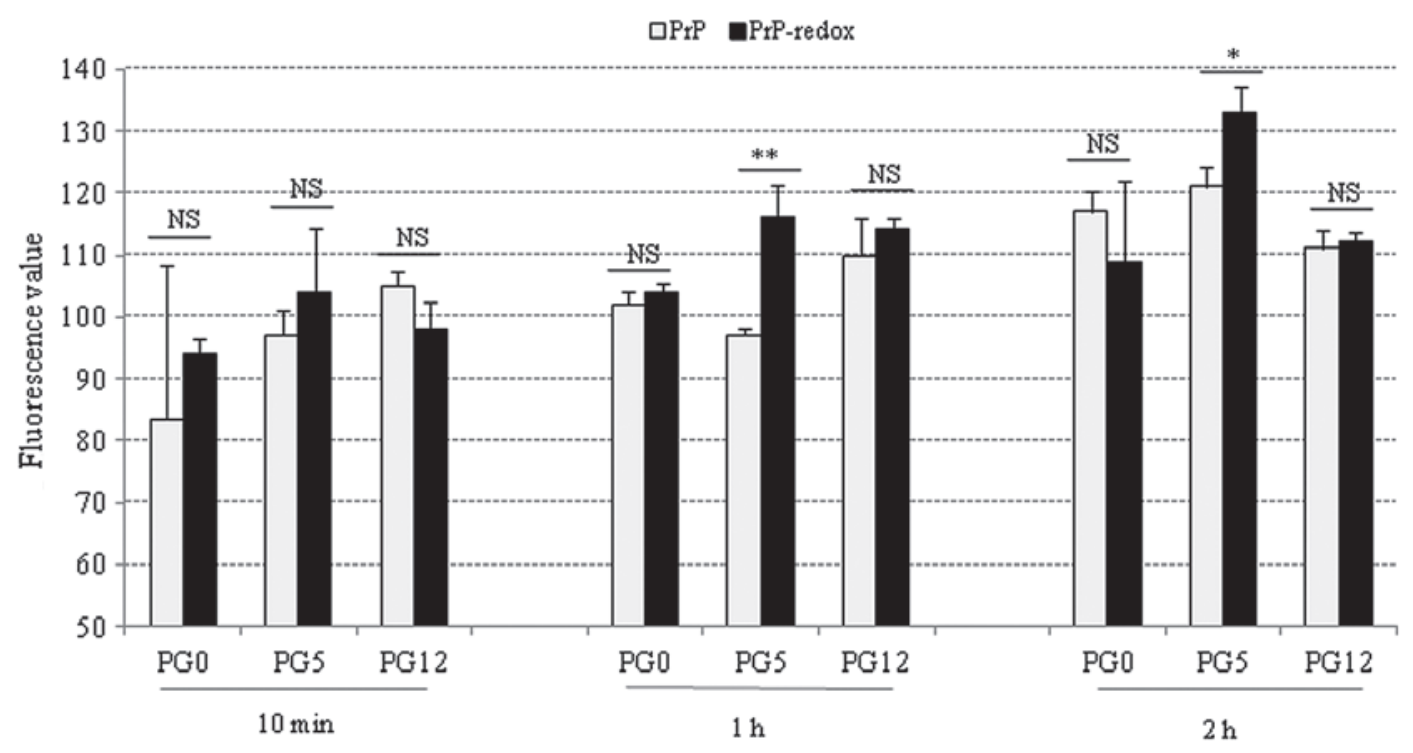

Figure 3. Thioflavin T assays of PG0, PG5 and PG12 PrPs prior to and following redox. Fluorescence values were measured from three independent experiments and are presented as the means \pm standard deviation. ${ }^{* *} \mathrm{P}<0.01$ and ${ }^{*} \mathrm{P}<0.05$. NS, not significant; PrP, prion protein.

increase in random-coil content; however, the increase in $\beta$-sheet content was limited (Table I).

Effects of redox on the fibril formation of the three recombinant human PrPs. To determine the potential effects of redox on PrP fibril formation, various PrP proteins were subjected to ThT assays prior to and following redox. Following incubation in assembling buffer for $10 \mathrm{~min}$, or 1 and $2 \mathrm{~h}$, the fluorescence value of each sample was measured. Generally, the fluorescence values of all tested PrP samples were increased along with prolonging the incubation times. In the 10-min preparations, redox-treated PG0 and PG5 exhibited slightly higher fluorescence values compared with in the untreated PrPs; however, none of these results were significant (Fig. 3, left panel). Redox-treated PG5 exhibited a significantly higher fluorescence value compared with in untreated PG5 at 1 and $2 \mathrm{~h}$, whereas no significance was detected in PG0 and PG12 PrPs between the redox-treated and untreated groups at 1 and $2 \mathrm{~h}$ (Fig. 3, middle and right panels). These results indicated that redox may increase fibril formation of wild-type PrP, but may not affect the fibril formation of mutated PrPs with insertion or deletion of octarepeats.

Effects of redox on $P K$ resistance of the three recombinant human PrPs. To evaluate the effects of redox on PK resistance, three PrP constructs were digested with various doses of PK before and after redox, and the reactions were terminated at $20 \mathrm{~min}$ or $1 \mathrm{~h}$ post-digestion. All samples were subjected to PrP-specific western blot analyses and the gray values were normalized to those of the PrP without PK $(0 \mu \mathrm{g})$. In the preparations digested for $20 \mathrm{~min}$ (Fig. 4A, left panel), residual full-length PrP signals were observed in redox-treated and untreated PG0 at the lowest PK dosage $(6.25 \mu \mathrm{g})$, redox-treated PG12 (6.25 and 12.5 $\mu \mathrm{g}$ PK) and redox-untreated PG12 (6.25 $\mu \mathrm{g}$ $\mathrm{PK})$, but not in PG5 reactions. Analysis of the degraded PrP in the blots revealed that redox-treated PG5 contained more residual signals than untreated PG5. In the preparations digested for $1 \mathrm{~h}$ (Fig. 4B), the PrP signal almost disappeared in $\mathrm{PG} 5$ reactions, with the exception of an extremely weak residual signal in redox-treated PG5 following treatment with the lowest dose of PK $(6.25 \mu \mathrm{g})$. Residual full-length PrP was still detectable in PG0 reactions $(6.25 \mu \mathrm{g} \mathrm{PK})$ with or without redox; however, the degraded PrP signals were reduced in untreated PG0 following treatment with $12.5 \mu \mathrm{g}$ PK compared with in the redox-treated PG0. Notably, more full-length PrP signals were observed in redox-treated PG1 2 compared with in untreated PG12 following treatment with $6.25 \mu \mathrm{g} \mathrm{PK}$, and PrP signals were detectable in redox-treated PG12 only following treatment with $12.5 \mu \mathrm{g}$ PK. These data indicated that redox can increase PK resistance of wild-type and mutated recombinant human PrPs. The PrP mutants with insertion or deletion of octarepeats possessed stronger PK-resistant activities.

\section{Discussion}

The formation of disulfide bridges is one of numerous types of post-translational protein modification. Proteins in which 

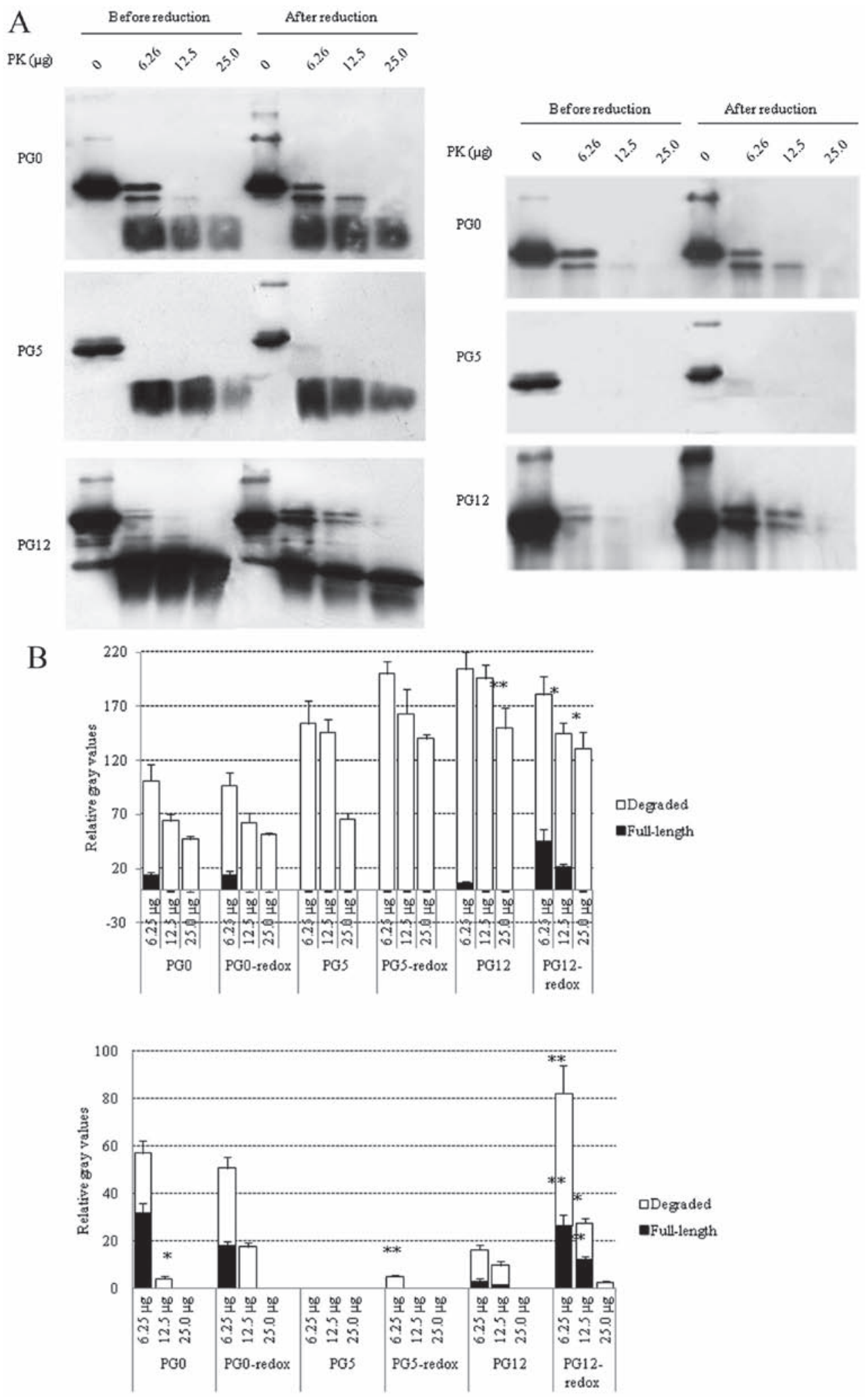

Figure 4. PK resistance of PG0, PG5 and PG12 before and after redox. (A) Western blot analyses of various PrPs digested with different concentrations of PK for 20 min (left panel) or $1 \mathrm{~h}$ (right panel). (B) Semi-quantitative analyses of the average gray values of the PK-resistant residual signals of various PrPs digested with different concentrations of PK for $20 \mathrm{~min}$ (upper panel) or $1 \mathrm{~h}$ (lower panel). Each gray value of PK-resistant residual signals was normalized to that of input PrP (designated as 100). Data are presented as the means \pm standard deviation. Full-length: The residual signal mobilizing at the same position of individual full-length PrP. Degraded: Degraded PrP residual signal mobilizing at a lower position than individual full-length PrP. Statistical analysis was performed between individual PrP constructs before and after redox, and marked above the redox-treated $\operatorname{PrP}$ columns. ${ }^{* *} \mathrm{P}<0.01$ and ${ }^{*} \mathrm{P}<0.05$. PK, proteinase $\mathrm{K}$; PrP, prion protein.

two cysteines have formed a disulfide bond are oxidized, whereas proteins without disulfide bonds are reduced, or are known to be in the thiol state (15). A previous study verified that the conformational structures, chemical characteristics 
and biological functions of various proteins may be markedly altered during the transformation between these two states (16). The present study hypothesized that following redox, the three recombinant human PrPs would exhibit reduced $\alpha$-helix content and increased PK resistance. The results of the present study demonstrated that redox produced distinct effects on the different PrP constructs. These findings indicated that the number of octarepeats in PrP affects its biochemical features.

Under the experimental conditions, wild-type $\operatorname{PrP}$ was revealed to be more sensitive to redox compared with the two PrP mutants. In addition to the reduction in $\alpha$-helix content and the increase in PK resistance, oxidized wild-type PrP contained increased $\beta$-sheet content, and easily formed aggregates and fibrils. The biochemical phenotype of normal PrP can be influenced by numerous small molecules, including metal ions (17). Exposure of recombinant human PrP to $\mathrm{Mn}^{2+}$ increases $\beta$-sheet content and enhances the formation of aggregates in vitro (18). Furthermore, saturation of recombinant mouse $\operatorname{PrP}$ with $\mathrm{Cu}^{2+}$ has been reported to efficiently enhance conversion to PK-resistant PrP in protein misfolding cyclic amplification (19). Notably, in a previous study, the biochemical features of oxidized PG5, formed via redox, share similarity with $\mathrm{Cu}^{2+}$ - and $\mathrm{Mn}^{2+}$-treated PrP, even with pathological $\mathrm{PrP}^{\mathrm{Sc}}$ (20). Therefore, it may be hypothesized that, as the propagating substrate for prions, increased oxidation of $\operatorname{PrP}^{\mathrm{C}}$ may benefit the conversion from $\operatorname{PrP}^{\mathrm{C}}$ to $\mathrm{PrP}^{\mathrm{Sc}}$. Further evaluation of the redox state in the microenvironment of the central nervous system during prion infection may help to improve understanding of prion biology.

Although the crystal structure of pathological $\mathrm{PrP}^{\mathrm{Sc}}$ remains to be elucidated, it is well-known that the $\operatorname{PrP}^{\mathrm{Sc}}$ molecule is rich in $\beta$-sheet. Alterations to the conformational structures, e.g., a reduction in $\beta$-sheet content and dissociation of prion rods, may reduce, and even remove, prion infectivity (21). It has been suggested that changes to covalent bonds occur during conversion to $\mathrm{PrP}^{\mathrm{Sc}}$, besides conformational changes (22). PrP has the ability to self-aggregate and form oligomers, which can be observed in vitro and in vivo (23). Increased amounts of the oligomeric form of PrP can be achieved via redox on the thiol group within the PrP peptide, thus highlighting the importance of disulfide bond formation in this activity (24). Furthermore, the reduction and alkylation of PrP in vitro has been proposed to inhibit conversion to $\mathrm{PrP}^{\mathrm{Sc}}$. Using the alkylating antitumor drug, mechlorethamine, prion replication in vitro is efficiently inhibited (25), which may indicate that formation of the $\operatorname{PrP}$ oligomer, and even $\mathrm{PrP}^{\mathrm{Sc}}$, depends on formation of an intermolecular disulfide bond.

Maintenance of the correct number of octarepeats in $\operatorname{PrP}$ is critical for protein functions. Insertion or deletion of octarepeats in the PrP gene is directly associated with inherited human prion diseases. Our previous study confirmed that the molecular interaction of PrP with tubulin depends on octarepeats (26). In addition, although the binding activity of PrP to tubulin is closely associated with the number of octarepeats, the regulation on microtubule polymerization is also associated with the number of octarepeats; mutants which exhibited strong inhibition on microtubule polymerization (27). The neuroprotective effects of PrP are also associated with the number of octarepeats, both insertions and deletions of octarepeats exert marked cytotoxic effects (28). In the present study, compared with wild-type PG5, PG0 and PG12 exhibited very limited alterations in sedimenta- tion and fibril formation, and increased random-coil content following redox. However, whether the octarepeat region affects the formation of a disulfide bond during redox under these experimental conditions remains unclear. The distinct outcomes detected among the various PrP constructs after redox emphasized the importance of the correct number of octarepeats within PrP with regards to its biological features.

Under the experimental conditions of the present study, PG0 and PG12 maintained solubility after redox. Coincidentally, the formation of fibrils in oxidized PG0 and PG12 was not increased; the exact reason for this is currently unknown. CD spectra detected marked increases in the random-coil content of redox-treated PG0 and PG12. Furthermore, $\beta$-sheet content was reduced in redox-treated PG0 and exhibited little change in redox-treated PG12. Those secondary structural alterations in PG0 and PG12 following redox may be associated with the detected biochemical phenotypes.

In accordance with previous data (29), the PK resistance of PG0 and PG12 was much stronger than PG5 in the present study. After redox, the PK resistance of oxidized PG5 and PG12 was markedly increased, whereas that of oxidized PG0 was only slightly increased. $\mathrm{PK}$ resistance of $\mathrm{PrP}^{\mathrm{Sc}}$ is believed to be associated with an increase in $\beta$-sheet content; therefore, the redox-induced increase in $\beta$-sheet content and decrease in $\alpha$-helix content in oxidized PG5 may be associated with its increased PK resistance. Conversely, the increases in PK resistance of PG12 and PG0 seem to not be directly associated with an increase in $\beta$-sheet content, since redox did not induce a significant increase in $\beta$-sheet in PG12, and $\beta$-sheet content was reduced in PG0 following redox. These findings indicated that besides detectable alterations in the secondary structure of PrP because of formation of disulfide bond, other unknown conformational changes associated with an alteration in the number of octarepeats may be involved in the appearance of PK resistance in PrP mutants. This partly indicates the treatment direction of genetic CJD.

\section{Acknowledgements}

The present study was supported by the Chinese National Natural Science Foundation Grants (grant nos. 81630062 and 81572048), the China Mega-Project for Infectious Disease (grant nos. 2011ZX10004-101 and 2012ZX10004215) and the SKLID Development Grant (grant nos. 2015SKLID503 and 2016SKLID603).

\section{Competing interests}

The authors declare that they have no competing interests.

\section{References}

1. Colby DW and Prusiner SB: Prions. Cold Spring Harb Perspect Biol 3: a006833, 2011.

2. Prusiner SB, Scott MR, DeArmond SJ and Cohen FE: Prion protein biology. Cell 93: 337-348, 1998.

3. Stellato F, Minicozzi V, Millhauser GL, Pascucci M, Proux O, Rossi GC, Spevacek A and Morante S: Copper-zinc cross-modulation in prion protein binding. Eur Biophys J 43: 631-642, 2014.

4. Paucar M, Xiang F, Moore R, Walker R, Winnberg E and Svenningsson P: Genotype-phenotype analysis in inherited prion disease with eight octapeptide repeat insertional mutation. Prion 7: 501-510, 2013. 
5. Beck JA, Mead S, Campbell TA, Dickinson A, Wientjens DP, Croes EA, Van Duijn CM and Collinge J: Two-octapeptide repeat deletion of prion protein associated with rapidly progressive dementia. Neurology 57: 354-356, 2001

6. Wiseman FK, Cancellotti E, Piccardo P, Iremonger K, Boyle A, Brown D, Ironside JW, Manson JC and Diack AB: The glycosylation status of $\mathrm{PrP}^{\mathrm{C}}$ is a key factor in determining transmissible spongiform encephalopathy transmission between species. J Virol 89: 4738-4747, 2015

7. Yang Y, Chen L, Pan HZ, Kou Y and Xu CM: Glycosylation modification of human prion protein provokes apoptosis in HeLa cells in vitro. BMB Rep 42: 331-337, 2009.

8. Kuczius T and Kelsch R: Effects of metal binding on solubility and resistance of physiological prions depend on tissues and glycotypes. J Cell Biochem 114: 2690-2698, 2013.

9. Ning L, Guo J, Jin N, Liu $\mathrm{H}$ and Yao X: The role of Cys179-Cys214 disulfide bond in the stability and folding of prion protein: Insights from molecular dynamics simulations. J Mol Model 20: 2106, 2014.

10. Maiti NR and Surewicz WK: The role of disulfide bridge in the folding and stability of the recombinant human prion protein. J Biol Chem 276: 2427-2431, 2001.

11. Singh N, Singh A, Das D and Mohan ML: Redox control of prion and disease pathogenesis. Antioxid Redox Signal 12: 1271-1294, 2010.

12. An R, Dong C, Lei Y, Han L, Li P, Chen J, Wang G, Shi Q, Gao C, Jiang $\mathrm{H}$, et al: PrP mutants with different numbers of octarepeat sequences are more susceptible to the oxidative stress. Sci China C Life Sci 51: 630-639, 2008.

13. Zhang FP, Zhang J, Zhou W, Zhang BY, Hung T and Dong XP: Expression of $\operatorname{PrP}(C)$ as HIS-fusion form in a baculovirus system and conversion of expressed PrP-sen to PrP-res in a cell-free system. Virus Res 87: 145-153, 2002.

14. Lee S and Eisenberg D: Seeded conversion of recombinant prion protein to a disulfide-bonded oligomer by a reduction-oxidation process. Nat Struct Biol 10: 725-730, 2003.

15. Dietz KJ and Hell R: Thiol switches in redox regulation of chloroplasts: Balancing redox state, metabolism and oxidative stress. Biol Chem 396: 483-494, 2015.

16. Ckless K: Redox proteomics: From bench to bedside. Adv Exp Med Biol 806: 301-317, 2014.

17. Rana A, Gnaneswari D, Bansal S and Kundu B: Prion metal interaction: Is prion pathogenesis a cause or a consequence of metal imbalance? Chem Biol Interact 181: 282-291, 2009.

18. Zhu F, Davies P, Thompsett AR, Kelly SM, Tranter GE, Hecht L, Isaacs NW, Brown DR and Barron LD: Raman optical activity and circular dichroism reveal dramatic differences in the influence of divalent copper and manganese ions on prion protein folding. Biochemistry 47: 2510-2517, 2008.
19. Kim NH, Choi JK, Jeong BH, Kim JI, Kwon MS, Carp RI and Kim YS: Effect of transition metals $(\mathrm{Mn}, \mathrm{Cu}, \mathrm{Fe})$ and deoxycholic acid (DA) on the conversion of $\mathrm{PrP}^{\mathrm{C}}$ to PrPres. FASEB J 19: 783-785, 2005.

20. Cingaram PK, Nyeste A, Dondapati DT, Fodor E and Welker E: Prion protein does not confer resistance to hippocampus-derived zpl cells against the toxic effects of $\mathrm{Cu}^{2+}, \mathrm{Mn}^{2+}, \mathrm{Zn}^{2+}$ and $\mathrm{Co}^{2+}$ not supporting a general protective role for $\mathrm{PrP}$ in transition metal induced toxicity. PLoS One 10: e0139219, 2015.

21. Riesner D, Kellings K, Post K, Wille H, Serban H, Groth D, Baldwin MA and Prusiner SB: Disruption of prion rods generates 10-nm spherical particles having high alpha-helical content and lacking scrapie infectivity. J Virol 70: 1714-1722, 1996.

22. Lu BY and Chang JY: Rapid and irreversible reduction of protein disulfide bonds. Anal Biochem 405: 67-72, 2010.

23. Yuan Z, Yang L, Chen B, Zhu T, Hassan MF, Yin X, Zhou X and Zhao D: Protein misfolding cyclic amplification induces the conversion of recombinant prion protein to PrP oligomers causing neuronal apoptosis. J Neurochem 133: 722-729, 2015

24. Shin JY, Shin JI, Kim JS, Yang YS, Shin YK, Kim KK, Lee S and Kweon DH: Disulfide bond as a structural determinant of prion protein membrane insertion. Mol Cells 27: 673-680, 2009.

25. Zhou X, BiH, Wong J, Shimoji M, Wang Y, Yuan J, Xiao X, Wang GX and Zou WQ: Alkylating antitumor drug mechlorethamine conceals a structured PrP domain and inhibits in vitro prion amplification. J Toxicol Environ Health A 74: 1493-1503, 2011.

26. Dong CF, Wang XF, An R, Chen JM, Shan B, Han L, Lei YJ, Han J and Dong XP: Interaction analysis between various PrP fusion proteins and the tubulin in vitro. Bing Du Xue Bao 23: 28-32, 2007 (In Chinese).

27. Dong CF, Shi S, Wang XF, An R, Li P, Chen JM, Wang X, Wang GR, Shan B, Zhang BY, et al: The N-terminus of PrP is responsible for interacting with tubulin and fCJD related PrP mutants possess stronger inhibitive effect on microtubule assembly in vitro. Arch Biochem Biophys 470: 83-92, 2008.

28. Mitteregger G, Vosko M, Krebs B, Xiang W, Kohlmannsperger V, Nölting S, Hamann GF and Kretzschmar HA: The role of the octarepeat region in neuroprotective function of the cellular prion protein. Brain Pathol 17: 174-183, 2007.

29. Li XL, Dong CF, Wang GR, Zhou RM, Shi Q, Tian C, Gao C, Mei GY, Chen C, Xu K, et al: Manganese-induced changes of the biochemical characteristics of the recombinant wild-type and mutant PrPs. Med Microbiol Immunol (Berl) 198: 239-245, 2009. 